\title{
Non-melanoma skin cancer treated with HDR Valencia applicator: clinical outcomes
}

\author{
Alejandro Tormo, MD', Francisco Celada, MD!', Silvia Rodriguez, MD², Rafael Botella, MD³, Antonio Ballesta, MD', \\ Michael Kasper, MD4, Zoubir Ouhib, PhD", Manuel Santos, MD², Jose Perez-Calatayud, PhD2,5 \\ IRadiotherapy Department, La Fe University and Polytechnic Hospital, Valencia, Spain, 2Radiotherapy Department, Benidorm Hospital \\ Alicante, Spain, ${ }^{3}$ Dermatology Department, La Fe University and Polytechnic Hospital, Valencia, Spain, ${ }^{4}$ Radiation Oncology, Lynn Regional \\ Cancer Center, Boca Raton, Florida, USA, ${ }^{5}$ Physics Section, Department of Radiation Oncology, La Fe University Hospital, Valencia, Spain
}

\begin{abstract}
Purpose: Radiotherapy (RT) has played a significant role in treating non melanoma skin cancer (NMSC). Highdose-rate brachytherapy (HDR-BT) approaches have a paramount relevance due to their adaptability, patient protection, and variable dose fractionation schedules. Several innovative applicators have been introduced to the brachytherapy community. The Valencia applicator is a new superficial device that improves the dose distribution compared with the Leipzig applicator. The purpose of this work is to assess the tumor control, cosmesis, and toxicity in patients with NMSC treated with the Valencia applicator and a new regimen of hypofractionation.

Material and methods: From January 2008 to March 2010, 32 patients with 45 NMSC lesions were treated with the Valencia applicator in the Hospital La Fe. The gross tumor volume was visually assessed, but the tumor depth was evaluated using ultrasound imaging. All lesions for the selected cases were limited to $4 \mathrm{~mm}$ depth. The prescription dose was $42 \mathrm{~Gy}$ in 6 or 7 fractions (biologically effective dose [BED] $\approx 70 \mathrm{~Gy}$ ), delivered twice a week.

Results: Ninety-eight percent of the lesions were locally controlled at 47 months from treatment. Ninety-three percent of patients were out at least 36 months from treatment. The treatment was well tolerated in all cases. The highest skin toxicity was grade 1 RTOG/EORTC, having resolved with topical treatment at 4 weeks in all but one case which required 2 months. There were no grade 2 or higher late adverse events.

Conclusions: In patients with superficial basal cell carcinoma lesions less than $25 \mathrm{~mm}$ in maximum diameter, HDRBT treatment with the Valencia applicator using a hypofractionated regimen provides excellent results, for both cosmetic and local control at a minimum of 3 years follow-up. Moreover, the shorter hypofractionated regimen facilitates compliance, which is very relevant for the elderly patients in our series. Valencia applicators offer a simple, safe, quick, and attractive nonsurgical treatment option.
\end{abstract}

Key words: HDR, skin brachytherapy, skin cancer, Valencia applicators.

\section{Purpose}

Non melanoma skin cancer (NMSC) is the most common malignancy and its incidence has been reported in the last years to be increasing rapidly, even in young populations [1]. It affects 2-3 million people each year in the United States [2], and a similar incidence is expected in Europe. Although, NMSC is usually not a life threatening condition, it is a growing public health concern because of the significant impact on quality of life [2-4]. Surgery, cryotherapy, topical chemotherapy, photodynamic therapy, and radiotherapy (RT) are treatments options for NSMC.

Surgery (Mohs or excision) is the most frequently offered treatment. For many decades, RT has played a significant role in treating NMSC [5]. Superficial X-rays, electron beam, megavoltage photons, and low- (LDR) or high-doserate (HDR) brachytherapy (BT) have been employed with success. There are also new emerging techniques such as electronic brachytherapy (EBT) [6]. In general, treatment options are chosen based upon institutional resources and the experiences of various specialists. With the incorporation of high-dose-rate brachytherapy (HDR-BT) and remote afterloaders in the 1960's, there has been a renewed interest in BT. High-dose-rate brachytherapy approaches have a paramount relevance due to adaptability, patient protection, and variable dose fractionations schedules, which achieve excellent cures rates and cosmetic results [7-9]. Several innovative applicators have been introduced to the BT community and the use of skin BT has increased significantly. The Valencia applicator is a new superficial
Address for correspondence: Jose Perez-Calatayud, PhD, Radiotherapy Department, La Fe University Hospital, Valencia, Spain, Avda Bulevar s/n 46026 Valencia, Spain, phone +34 961245103 ,

• e-mail: perez_jos@gva.es
Received: 27.12 .2013

Accepted: 18.02.2014

Published: 26.06.2014 
device with improved dose distribution when compared to the Leipzig applicator $[10,11]$. The Valencia $[10,11]$ applicators were designed to be used with the HDR afterloader microSelectron v2 (Nucletron, an Elekta company, Elekta AB, Stockholm, Sweden), and come in two useful sizes: 2 and $3 \mathrm{~cm}$ diameters. These cup-shaped applicators limit the irradiation to the treated area using tungsten-alloy shielding. The design of these applicators was based on the Leipzig applicators [12-15] with the addition of a flattening filter to improve the dose profiles and limit the penumbra. This allows focusing the radiation on the target while normal tissue irradiation is minimized.

The purpose of this study is to assess the tumor control, cosmesis, and toxicity events in patients with basal cell carcinoma (BCC) treated with the Valencia applicator using a new regimen of hypofractionation.

\section{Material and methods}

\section{Eligibility and study design}

From January 2008 to March 2010, 32 patients with 45 NMSC lesions were treated with the Valencia applicators in the Radiotherapy Department of Hospital La Fe, Valencia, Spain.

Patients enrolled in this study had a biopsy confirmed BCC located anywhere on the body or face except eyelids, and all cases presented as primary lesions. Initially, the maximum diameter accepted was 24 . In patients with multiple lesions, all lesions were treated concurrently. All patients were over 18 years old, but no other age restrictions were applied. Data were collected retrospectively.

\section{Treatment protocol}

The protocol used for these patients was developed with the Lynn Cancer Institute of Boca Raton Regional Hospital (Boca Raton, Florida), Hospital La Fe (Valencia, Spain), and Hospital Clinica Benidorm (Benidorm, Alicante, Spain) [16]. The tumor depth was evaluated using ultrasound imaging. Ultrasounds images were performed with the Siemens Acuson S 2000 ultrasound system (Siemens AG, Munich, Germany), using a $19 \mathrm{mHz}$ linear probe. To improve imaging, a gel pad was systematically used. The acquisition was done by longitudinal and transversal planes, evaluating the echogenicity and thickness of both skin and subcutaneous cellular tissue at lesion lev$\mathrm{el}$, measuring the lesion depth. All lesions for the selected cases were limited to a maximum of $4 \mathrm{~mm}$ depth. This limitation was necessary to keep the skin dose at acceptable levels, since the percent depth dose of the ${ }^{192}$ Ir Valencia applicators has a gradient of about $10 \%$ per mm [10].

The gross tumor volume (GTV) was generally assessed visually. The appropriate Valencia applicator size (2 or $3 \mathrm{~cm}$ ) was chosen to allow for a minimum margin of $5 \mathrm{~mm}$ from the field edge. In the cases with tighter margins, the GTV was assessed by a dermatologist with a dermatoscope. The treatment dose was prescribed at $3 \mathrm{~mm}$ for lesions with depth of $3 \mathrm{~mm}$ or less, and at $4 \mathrm{~mm}$ for those between $3 \mathrm{~mm}$ and $4 \mathrm{~mm}$ in depth. High accuracy in tumor depth measurement is critical due to the high dose gradient. Thus, for a typical prescription to $3 \mathrm{~mm}$, the surface dose will reach approximately $130 \%$. The prescription dose was 42 Gy in 6 or 7 fractions (biological effective dose (BED) $\approx 70$ Gy), delivered two times a week, with a minimum interval of 48 hours between fractions, and not exceeding 3 weeks. Immobilization was achieved in most cases using an articulated arm device provided by Nucletron. Other methods of immobilization such as simple homemade arm, tape, or thermoplastic mask, when needed, were also occasionally used. Some examples are illustrated on Figure 1. Skin marker delineating the outside applicator circumference was used to ensure reproducible treatment conditions.

All treatments were delivered under the direct supervision of the radiation oncologist for accurate applicator positioning. Patients were instructed not to apply makeup or ointment prior to treatment.

\section{End points}

The end points chosen for this study were the analysis of the efficacy, safety outcomes, and cosmetic results of the treatment of NMSC with BT, using the Valencia applicators with a hypofractionated regimen. Acute and chronic toxicities were evaluated according to the Radiation Therapy Oncology Group (RTOG) and the European Organization for Research and Treatment of Cancer (EORTC) scale [17]. Patients were also consulted about their cosmetic impression using a survey with questions related to their satisfaction.

\section{Results}

\section{Patient and treatment characteristics}

Forty-five lesions in 32 patients were treated between January 2008 and March 2010. Although the age range
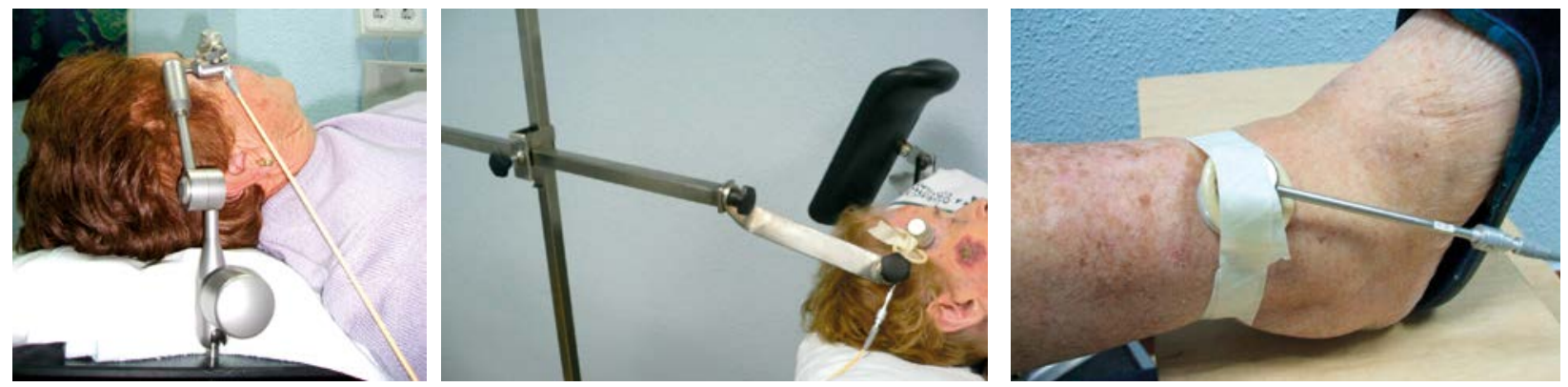

Fig. 1. Examples of treatment set-ups 
was wide (43-97 years), the majority $(70 \%)$ of these patients were over 70 years old. No recurrent lesions were included. Histology was BCC in all lesions. As usual in BCC [18], most $(86 \%)$ lesions were located on the head. The median maximum diameter of the lesion was $10 \mathrm{~mm}$ (range: 3-25). Only two lesions had a depth greater than $3 \mathrm{~mm}(4 \mathrm{~mm})$. Table 1 summarizes patient and lesion characteristics. Lesions were treated with a total dose of $42 \mathrm{~Gy}$, using 6 fractions (7 Gy/fraction; twice weekly) in $96 \%$ of the cases, and 7 fractions (6 Gy/fraction; twice weekly) in the remaining $4 \%$. The biological effective dose (BED), for both schemes is approximately 70 Gy $\operatorname{BED}(\alpha / \beta: 10)$. The treatment was prescribed at $3 \mathrm{~mm}$ depth in 46 lesions and at $4 \mathrm{~mm}$ in 2 lesions. Median lesion diameter was $10 \mathrm{~mm}$, ranged 3-25, median depth $2 \mathrm{~mm}$, ranged 0-4. We used 25 applicators size 2 (55.5\%) and 20 applicators size $3(44.5 \%)$.

The median follow-up was 47 months (range: $31-60$ ). In $93 \%$ of the patients, follow-up was 36 months or more.

\section{Efficacy results}

Ninety-eight percent of the lesions completely regressed. Examples of clinical results are shown in Figure 2. At 3 months, only one lesion persisted and it was centrally located. This patient had an extra-facial BCC. Tumor depth of this lesion was originally assessed in the ultrasound as $2 \mathrm{~mm}$. Following the protocol, the depth used for the dose prescription was $3 \mathrm{~mm}$. After subsequent review of the ultrasound images, it was established that tumor depth was actually greater than $3 \mathrm{~mm}$. No other recurrences were detected after a minimum follow-up of 31 months.

\section{Adverse events and cosmetic results}

The treatment was well tolerated in all cases. The highest skin toxicity was grade 1 RTOG/EORTC [17], having resolved with topical treatment at 4 weeks in all but one case, which required 2 months. There were no grade 2 or higher late adverse events. When patients were consulted about their cosmetic impression, all except one (a young woman with hypopigmentation at abdominal location) were very satisfied.

\section{Discussion}

Surgery is often the primary treatment for a NMSC lesion due to its reported low rates of recurrence $[19,20]$. Typically, RT is an option for lesions located in areas where surgery might be accompanied with functional or cosmetic deficits, for patients with comorbidities or surgical contraindications, and for the elderly. A wide variety of radiotherapy techniques including superficial $\mathrm{X}$-rays, orthovoltage $\mathrm{X}$-rays, megavoltage $\mathrm{X}$-rays, electron beam irradiation, low-dose-rate brachytherapy (LDR-BT), HDR-BT, and EBT are used. The term BT includes superficial and interstitial treatments, which selection depends of the tumour depth, volume to be treated, or both. Applicators in contact with the skin are used in cases of superficial and well circumscribed lesions. Commercially applicators like the Leipzig and Valencia applicators, as well as flaps
Table 1. Patient and lesion characteristics

\begin{tabular}{|c|c|c|}
\hline \multicolumn{3}{|l|}{ Demographics } \\
\hline Age (median) (years) & 78 & $43-97$ \\
\hline \multicolumn{3}{|l|}{ Gender } \\
\hline Male & 18 & $54.5 \%$ \\
\hline Female & 15 & $45.5 \%$ \\
\hline \multicolumn{3}{|l|}{ Histology } \\
\hline Basal cell carcinoma & 45 & $93 \%$ \\
\hline Bowen's disease & 3 & $7 \%$ \\
\hline \multicolumn{3}{|l|}{ Lesions per patient } \\
\hline 1 & 26 & $79 \%$ \\
\hline 2 & 3 & $9 \%$ \\
\hline 3 & 3 & $9 \%$ \\
\hline 7 & 1 & $3 \%$ \\
\hline \multicolumn{3}{|l|}{ Lesion location } \\
\hline Head & 39 & $82 \%$ \\
\hline Nose & 17 & $36 \%$ \\
\hline Face & 11 & $23 \%$ \\
\hline Scalp & 10 & $21 \%$ \\
\hline Ear & 1 & $2 \%$ \\
\hline Extremity & 5 & $10 \%$ \\
\hline Trunk & 4 & $8 \%$ \\
\hline
\end{tabular}

and moulds are available. Customized moulds made for individual cases with different materials are also utilized. In all cases, the treatment must be comfortable for the patient and easily reproducible to avoid uncertainties.

Currently, all of these approaches achieve high rates of tumour control when considering similar tumour and patient characteristics [21]. The published local control rates with HDR-BT vary from 72 to $100 \%$. Large differences between studies were identified and are related to follow-up time, total dose, dose per fraction, time between fractions, prescription depth, and types of applicators [22-31]. There are just a few publications of clinical results of HDR-BT with standardized superficial applicators. In 1999, Köhler-Brock et al. [7] published the results at 10 years of 520 patients treated with Leipzig applicators up to 30-40 Gy at 5-10 Gy/fr. The failure rate was $8 \%$, but Kaposi's sarcoma, melanomas, and skin metastases were included. Gahly et al. [32] and Gauden et al. [27] presented their experience with Leipzig and GM11004590 applicator, achieving complete remission in $95 \%$ and $97 \%$ of the cases, respectively, including primary and recurrent NMSC lesions (median follow-up 18 and 37 months, respectively).

This study is the first one that reports clinical results with the Valencia applicators. Although the size of the 
Before
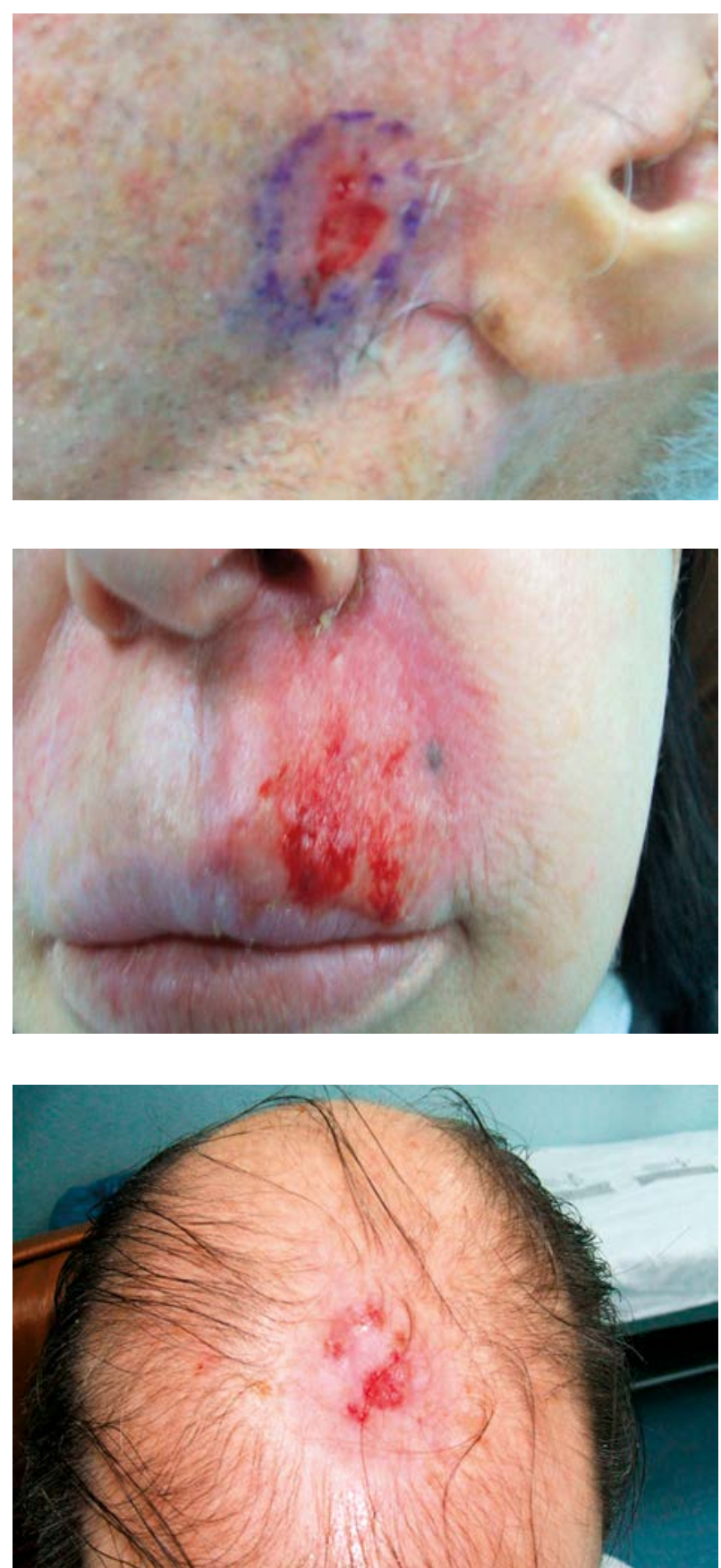

After
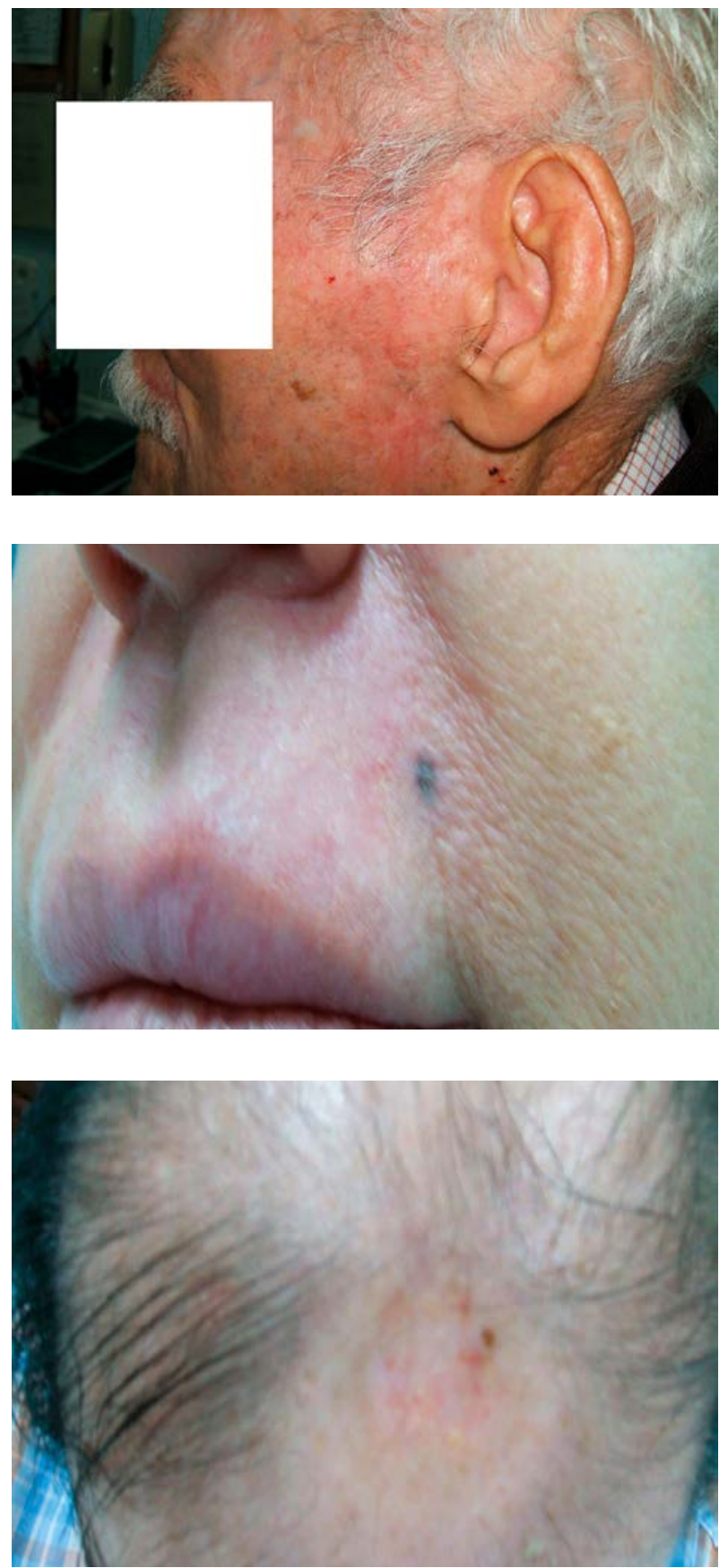

Fig. 2. Some examples of clinical results, before and after treatment

study is smaller than other HDR-BT publications, in our opinion it is important for several reasons. Firstly, the study population was very homogenous in tumour histology (BCC) and lesion size (less than $3 \mathrm{~cm}$ ). Secondly, all patients were treated in a highly consistent manner. Thirdly, the study also demonstrated safety and good tolerance in an older population (> 70 years). And finally, the local control was excellent, with mature follow-up. Performing HDR-BT with the Valencia applicators presents several advantages: dosimetric calculations are quick and safe, patient positioning and reproducibility are easy and comfortable. The hypofractionated regimen, $42 \mathrm{~Gy}$ in 6-7 fractions over 3 weeks or less, allows an ambulatory treatment that reduces costs, and facilitates compliance, which is essential in an elderly population as was treated in this series. In addition, the Valencia applicator using this hypofractionated regimen compares favourably with other regimens and techniques for efficacy as well as toxicity. The use of the Valencia applicators must be limited to superficial tumours (less than $4 \mathrm{~mm}$ depth) with a maximum diameter of $25 \mathrm{~mm}$ in order to guarantee adequate tumour coverage. The lateral homogeneity 
and flatness of the Valencia applicator is improved with respect to the Leipzig applicator and provides a smaller penumbra. The $80-20 \%$ penumbra values are significantly improved from the Leipzig $(6.2-9.5 \mathrm{~mm})$ to the Valencia applicators $(1.9 \mathrm{~mm})$. As a result, the useful treatment area of the Valencia applicator is larger. In 7 of the treated BCC lesions in this study, at least one of the margins was smaller than $5 \mathrm{~mm}$, but at least $3 \mathrm{~mm}$. In these 7 cases, the GTV was determined by dermatoscope. The other 35 lesions were treated with a minimum of a $5 \mathrm{~mm}$ margin. A recent publication based on a surgical series, suggests the following recommendations for CTV margins: $10 \mathrm{~mm}$ for BCC less than $2 \mathrm{~cm}, 13 \mathrm{~mm}$ for BCC larger than $2 \mathrm{~cm}, 11 \mathrm{~mm}$ for SCC less than $2 \mathrm{~cm}$, and $14 \mathrm{~mm}$ for SCC larger than $2 \mathrm{~cm}$ [33]. By contrast, a prospective study with 200 BCCs showed that $2 \mathrm{~mm}$ dermascopically detected excision margins achieved histologically confirmed complete excision in $98.5 \%$ of cases [34]. According to Brodland et al. [35], Mohs surgery margins of $4 \mathrm{~mm}$ for low risk SCC and $6 \mathrm{~mm}$ for high risk SCC are adequate to clear the tumor in $95 \%$ of cases. Based on the results of this and other BT studies, margins of $5 \mathrm{~mm}$ for BCC would appear adequate.

In our study, the only recurrence was centrally located. While the lesion depth was initially reported to be less than $3 \mathrm{~mm}$ according to the ultrasound study, a subsequent review of that image found that the depth was actually deeper. The treatment dose, in retrospect, was incorrectly prescribed to $3 \mathrm{~mm}$ depth. With the new data, the dose prescription would have been $4 \mathrm{~mm}$. While a possible solution to avoid undertreatment is to prescribe at $1 \mathrm{~mm}$ beyond the lesion depth, the high dose gradient ( $10 \%$ for each millimeter) has critical implications both for the coverage and for the surface dose. One of the most important limitations of the study is the toxicity data collection. Nevertheless, $98 \%$ of the skin toxicity resolved after 4 weeks with topical treatment. Regarding late toxicity, data interpretation is limited by the ambiguity of the scale used (RTOG/EORTC) and the subjective criterion of a unique data collector for each case. Although it may still be early for development of late toxicity, no findings were reported. For cosmesis, no scales were used, but all patients were consulted about their cosmetic impression. The results were favourable with the exception of one young patient with an abdominal lesion reported her dissatisfaction due to hypopigmentation. Using CTC v4.0 scale [36] this event could be classed as Grade 2 due to the associated psychosocial impact. All other patients were highly satisfied.

\section{Conclusions}

In patients with superficial BCC lesions less than $25 \mathrm{~mm}$ in maximum diameter, HDR-BT treatment with the Valencia applicator using a hypofractionated regimen provides excellent results, for both cosmetic and local control at a minimum of 3 years follow-up. Moreover, the shorter, hypofractionated regimen facilitates compliance, which is very relevant for the elderly patients in our series. Valencia applicators offer a simple, safe, quick, and attractive nonsurgical treatment option.

\section{Acknowledgements}

The authors wish to acknowledge all the staff at the Radiotherapy Department, Hospital La Fe and specially Dr. Facundo Ballester from the Valencia University for his review and comments.

\section{Disclosure}

Authors report no conflict of interest.

\section{References}

1. Wu TP, Stein JA. Skin Cancer in Young Women. J Drugs Dermatol 2013; 12: 568-572.

2. Rogers HW, Martin A. Incidence estimate of nonmelanoma skin cancer in the United States, 2006. Arch Dermatol 2010; 146: 283-287.

3. SkinCancerNet: What is skin cancer? Schaumberg, IL: American Academy of Dermatology. Available at: htp://www. skincarephysicians.com/skincancernet/whatis.html. Accessed March 17, 2012.

4. Lo A, Leonardi-Bee J, Bath-Hextall F. A systematic review of worldwide incidence of nonmelanoma skin cancer. $\mathrm{Br}$ J Dermatol 2012; 166: 1069-1080.

5. Lovett RD, Perez CA, Shapiro SJ et al. External radiation of epithelial skin cancer. Int J Radiat Oncol Biol Phys 1990; 19: 235-242.

6. Bhatnagar A. Nonmelanoma skin cancer treated with electronic brachytherapy: results at 1 year. Brachytherapy 2013; 12: 134-140.

7. Kohler-Brock A, Pragger W. The indications for and results of HDR afterloading therapy in diseases of the skin and mucosa with standardized surface applicators (the Leipzig Applicator). Strahlenther Onkol 1999; 175: 170-174.

8. Guix B, Finestres F, Tello J et al. Treatment of skin carcinomas of the face by high dose rate brachytherapy and custom made surface molds. Int J Radiat Oncol Biol Phys 2000; 47: 95-102.

9. Kowalik L, Lyczek J, Sawicki M et al. Individual applicator for brachytherapy for various sites of superficial malignant lesions. J Contemp Brachytherapy 2012; 5: 45-49.

10. Granero D, Perez-Calatayud J, Jimeno J et al. Design and evaluation of a HDR skin applicator with flattening filter. Med Phys 2008; 35: 495-503.

11. Granero D, Perez-Calatayud J, Ballester F et al. Radiation leakage study for the Valencia applicators. Physica Medica 2013; 29: 60-64.

12. Perez-Calatayud J, Granero D, Ballester F et al. A dosimetric study of the Leipzig applicators. Int J Rad Oncol Biol Phys 2005; 62: 579-584.

13. Niu H, His WC, Chu JCH et al. Dosimetric characteristics of the Leipzig surface applicators used in the high dose rate brachy radiotherapy. Med Phys 2004; 31: 3372-3377.

14. Hwang IM, Lin SY, Lin LC et al. Alternative effective modality of Leipzig applicator with an electron beam for the treatment of superficial malignancies. Nuc Inst Meth A 2003; 508: 460-466.

15. Evans MDC, Yassa M, Podgorsak EB et al. Surface applicators for high dose rate brachytherapy in aids-related Kapos1 sarcoma. Int J Radiat Oncol Biol Phys 1997; 39: 769-774.

16. Rodriguez S. $51^{\text {nd }}$ ASTRO Annual Meeting. Chicago. November 2009. Skin Cancer. Brachytherapy in Skin Cancer. Panel 8.

17. Cox JD, Stetz J, Pajak TF. Toxicity criteria of the Radiation Therapy Oncology Group (RTOG) and the European Organization for Research and Treatment of Cancer (EORTC). Int J Radiat Oncol Biol Phys 1995; 31: 1341-1346. 
18. Adam IR, Chen EH, Ratner D. Basal-Cell Carcinoma. N Engl J Med 2005; 353: 2262-2269.

19. Chren MM, Torres JS, Stuart SE et al. Recurrence after treatment of nonmelanoma skin cancer: A prospective cohort study. Arch Dermatol 2011; 147: 540-546.

20. MacFarlane L, Waters A, Evans A et al. Seven years' experience of Mohs micrographic surgery in a UK centre, and development of a UK minimum dataset and audit standards. Clin Exp Dermatol 2013; 8: 262-269.

21. Linos E, Parvataneni R, Stuart SE et al. Treatment of Nonfatal Conditions at the End of Life: Nonmelanoma Skin Cancer. JAMA Intern Med 2013; 173: 1006-1012.

22. Svoboda V, Kovarik J, Morris F. High dose-rate microselectron molds in the treatment of skin tumors. Int J Radiat Oncol Biol Phys 1995; 31: 967-972.

23. Allan E, Stanton A, Pye D et al. Fractionated high dose rate brachytherapy moulds: a precise treatment for carcinoma of the pinna. Radiother Oncol 1998; 48: 277-281.

24. Chan A, D'Souza O, Vujovic G et al. High dose rate (HDR) brachytherapy treatment for skin cancer: a novel approach. Int J Radiat Oncol Biol Phys 2003; 57 (2), Supplement.

25. Ghaly M, Birnes R, Musmacher J et al. HDR Brachytherapy with standardized surface applicators (the Leipzig applicator) as an alternative, radiotherapy treatment for superficial malignant skin lesions. Int J Radiat Oncol Biol Phys 2006; 66 (3) Supplement.

26. Fabrini MG, Perrone F, De Liguoro M et al. High dose rate brachytherapy in a large squamous cell carcinoma of the hand. Brachytherapy 2008; 7: 270-275.

27. Gauden S, Egan C, Pracy M. HDR brachytherapy for the treatment of skin cancers using standard surface applicators. Brachytherapy 2008; 7: s159 (abstract).

28. Montero A, Hernanz R, Capuz AB et al. High-dose-rate (HDR) plesiotherapy with custom-made moulds for the treatment of non-melanoma skin cancer. Clin Transl Oncol 2009; 11: 760-764.

29. Kanikowski M. HDR brachytherapy of skin cancer in material of Greater Poland Cancer Center. J Contemp Brachytherapy 2009; 3: 197 (abstract).

30. Maroñas M, Guinot JL, Arribas L et al. Treatment of facial cutaneous carcinoma with high dose rate contact brachytherapy with customized molds. Brachytherapy 2011; 10: 221-227.

31. Ozyar E, Gurdalli S. Mold brachytherapy can be an optional technique for total scalp irradiation. Int J Radiat Oncol Biol Phys 2002; 54: 1286.

32. Ghaly M, Zinkin H, Dannenberg M et al. HDR Brachytherapy with Standardized Surface Applicators in the Treatment of Superficial Malignant Skin Lesions. Int J Radiat Oncol Biol Phys 2008; 72: 505-506 (abstract).

33. Khan L, Choo R, Breen D et al. Recommendations for CTV margins in radiotherapy planning for non melanoma skin cancer. Radiother Oncol 2012; 104: 263-266.

34. Caresana G, Giardini R. Dermoscopy-guided surgery in basal cell carcinoma. J Eur Acad Dermatol Venereol 2010; 24: 13951399.

35. Brodland DG, Zitelli JA. Surgical margins for excision of primary cutaneous squamous cell carcinoma. J Am Acad Dermatol 1992; 27: 241-248.

36. National Cancer Institute Common Terminology Criteria for Adverse Events v4.0

37. NCI, NIH, DHHS. May 29, 2009. NIH publication \# 09-7473. http://ctep.cancer.gov/protocolDevelopment/electronic_ applications/ctc.htm 